\title{
Sonographic features of carotid artery dissection due to extension of aortic dissection: a case report
}

\author{
Christian Boßelmann ${ }^{1}(1)$ and Sven Poli ${ }^{*}$
}

\begin{abstract}
Background: Carotid artery dissection due to extension of aortic dissection (CAEAD) is a severe complication of acute aortic dissection. The risk of ischemic stroke is increased. Early sonographic detection and repeat evaluation are necessary to guide clinical management.
\end{abstract}

Case presentation: A 58-year-old male patient presents with sudden, tearing retrosternal pain. Point-of-care carotid ultrasound is used to establish the diagnosis of CAEAD. We describe a number of sonographic features and compare ultrasound to other imaging modalities.

Conclusions: Bedside carotid ultrasound enables rapid, sensitive and safe hemodynamic assessment, especially in critically ill patients.

Keywords: Neurology, Ultrasound, Point-of-care systems, Carotid artery dissection

\section{Background}

Aortic dissection (AD) is a clinical emergency. A tear in the tunica intima, the innermost layer of the vessel, is propagated by blood flowing into the false lumen. This dissection membrane can further extend into the aortic branches, including the carotid arteries. This is referred to as carotid artery extension of aortic dissection (CAEAD). Cerebral malperfusion can result from the dissection membrane restricting or occluding blood flow, akin to a carotid stenosis or large-vessel occlusion. Alternatively, artery-to-artery embolization or progressive stenosis from mural thrombosis may occur. Hence, rapid diagnosis and repeat hemodynamic evaluation are necessary to guide clinical management [1].

*Correspondence: sven.poli@uni-tuebingen.de

${ }^{2}$ Department of Neurology and Stroke, and Hertie-Institute for Clinical Brain Research, Eberhard-Karls University of Tübingen,

Hoppe-Seyler-Straße 3, 72076 Tübingen, Germany

Full list of author information is available at the end of the article

\section{Case presentation}

A 58-year-old male patient presented with sudden tearing retrosternal pain and dyspnea. No traumatic injury was reported. Chest computed tomography angiography (CTA) displayed an acute aortic dissection (Stanford type A, DeBakey type I), extending from the aortic sinus to both common iliac arteries and all supra-aortic branches (Fig. 1). The patient underwent emergency supracoronary ascending aorta replacement and aortic valve repair.

Postoperatively, dynamic evaluation through pulsedwave mode Doppler (8 MHz, vascular probe) and brightness-mode (B-mode) ultrasound demonstrated several sonographic features typical of a dissection. Notably, a dissection membrane was seen extending from the proximal right common carotid artery (CCA) to the distal ICA (Fig. 2, Additional file 1: Video S1). Flow direction in the true lumen was orthograde. No collateral flow was noted on transcranial Doppler, i.e., both the ophthalmic artery and the anterior cerebral artery were found to have normal, orthograde flow. Thus, distal high-grade stenosis or occlusion was excluded.

The left carotid arteries were also examined. On B-mode ultrasound, no dissection membrane or any of 


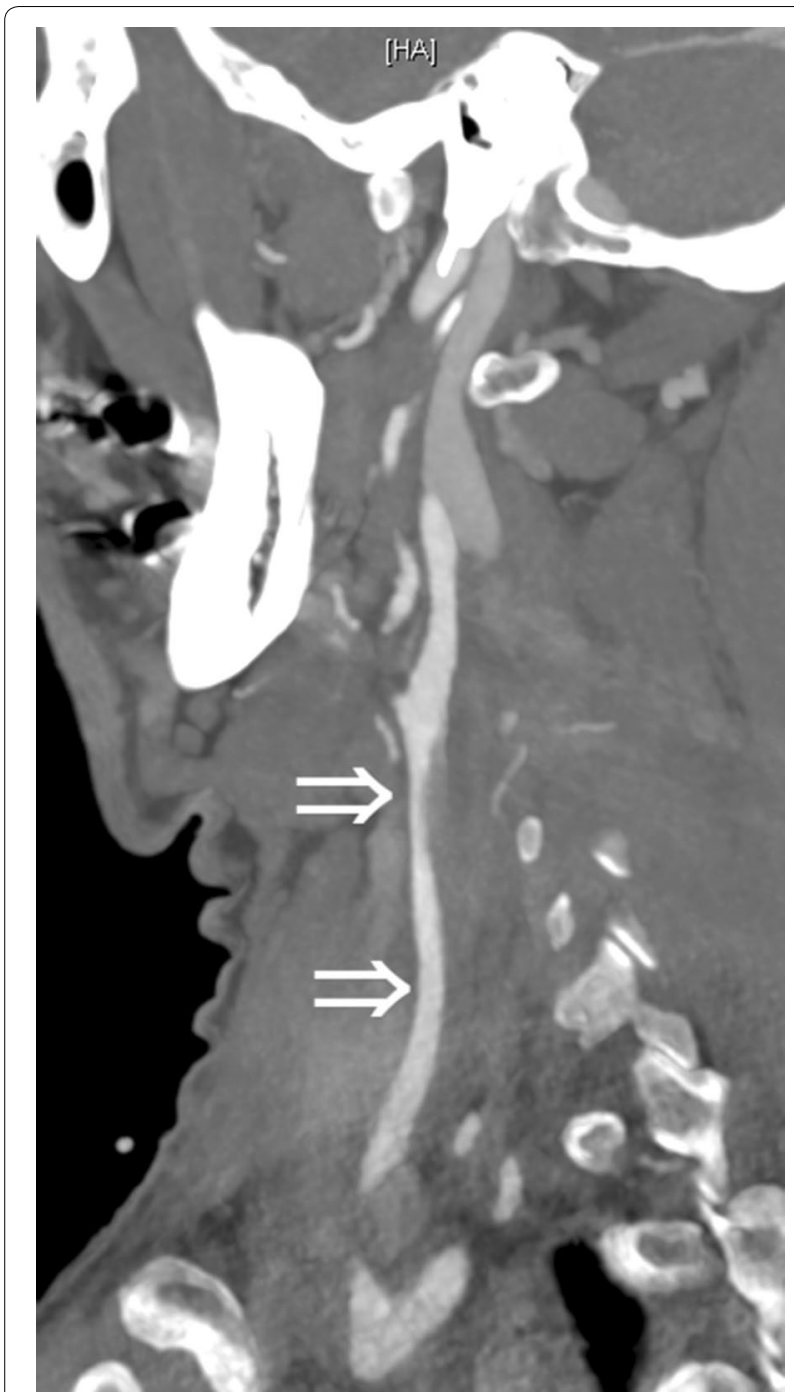

Fig. 1 Computed tomography angiography. On sagittal oblique maximum intensity projection (MIP), an extended and smoothly tapering stenosis of the right common carotid artery is seen (arrows)

the features described above were observed. However, Doppler ultrasound demonstrated a high peak systolic flow velocity of $470 \mathrm{~cm} / \mathrm{s}$ in the proximal left CCA (refer-

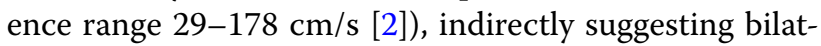
eral CCA dissection (not pictured).

\section{Discussion}

Here, we report a case where bedside carotid ultrasound enabled early detection and hemodynamic assessment of CAEAD in a critically ill patient. A number of sonographic features are shown, such as luminal stenosis, a perfused false lumen, and a dissection membrane.
Features not present include a thickened and hypoechogenic vessel wall, intracranial stenosis or occlusion, and pseudoaneurysm [3].

Four imaging modalities are primarily used in the diagnosis of carotid dissections. Digital subtraction angiography (DSA), i.e., contrast-enhanced invasive vascular imaging, remains the gold standard. Dynamic magnetic resonance angiography (MRA) may also be performed, especially in patients with iodinated contrast allergy or renal impairment. Fat-saturation sequences are useful to detect intramural hematoma, even if the lumen itself is not narrowed. Compared to DSA, MRA offers a variable performance at a sensitivity of $50-100 \%$ and a specificity of $29-100 \%$ [4] and is considered by the American Heart Association and the American Stroke Association to be the best initial screening test [5]. However, the speed of image acquisition is slow and MRA is not always feasible in intensive care unit (ICU) patients. Conversely, CTA is widely available, offers rapid image acquisition and compares favorably with DSA at a sensitivity of $64-100 \%$ and specificity of $67-100 \%$ [6].

Carotid ultrasound offers a number of advantages over static vessel imaging. Doppler mode yields information on flow characteristics including the peak systolic as well as diastolic flow velocities. This method is used to diagnose and grade stenosis and is especially valuable in the indirect assessment of the petrous ICA segment, where CTA is limited due to vessel wall calcifications or skull base artifacts. Using additional brightness-mode (B-mode) imaging with color-coded Doppler significantly increases the sensitivity in detecting carotid dissections, as compared to MRA, with no false-positive diagnoses [7]. Carotid ultrasound is also portable, which enables bedside examination and avoids unnecessary transports in ICU patients, which are associated with a number of serious adverse events [8]. Lastly, carotid ultrasound is the least invasive method, avoiding radiation exposure or the administration of a contrast agent. Hence, bedside carotid ultrasound is a safe and effective imaging modality in carotid dissections, suitable for use in critically ill patients.

Limitations may apply. Minor vessel damage, such as an intimal tear or small mural hematoma, may not be readily apparent on ultrasound. This applies especially to locations where B-mode imaging cannot be carried out, such as the skull base. Here, alternate imaging modalities, such as fat-saturation MRI or (contrastenhanced) MRA, may be useful as complementary diagnostic tools. 


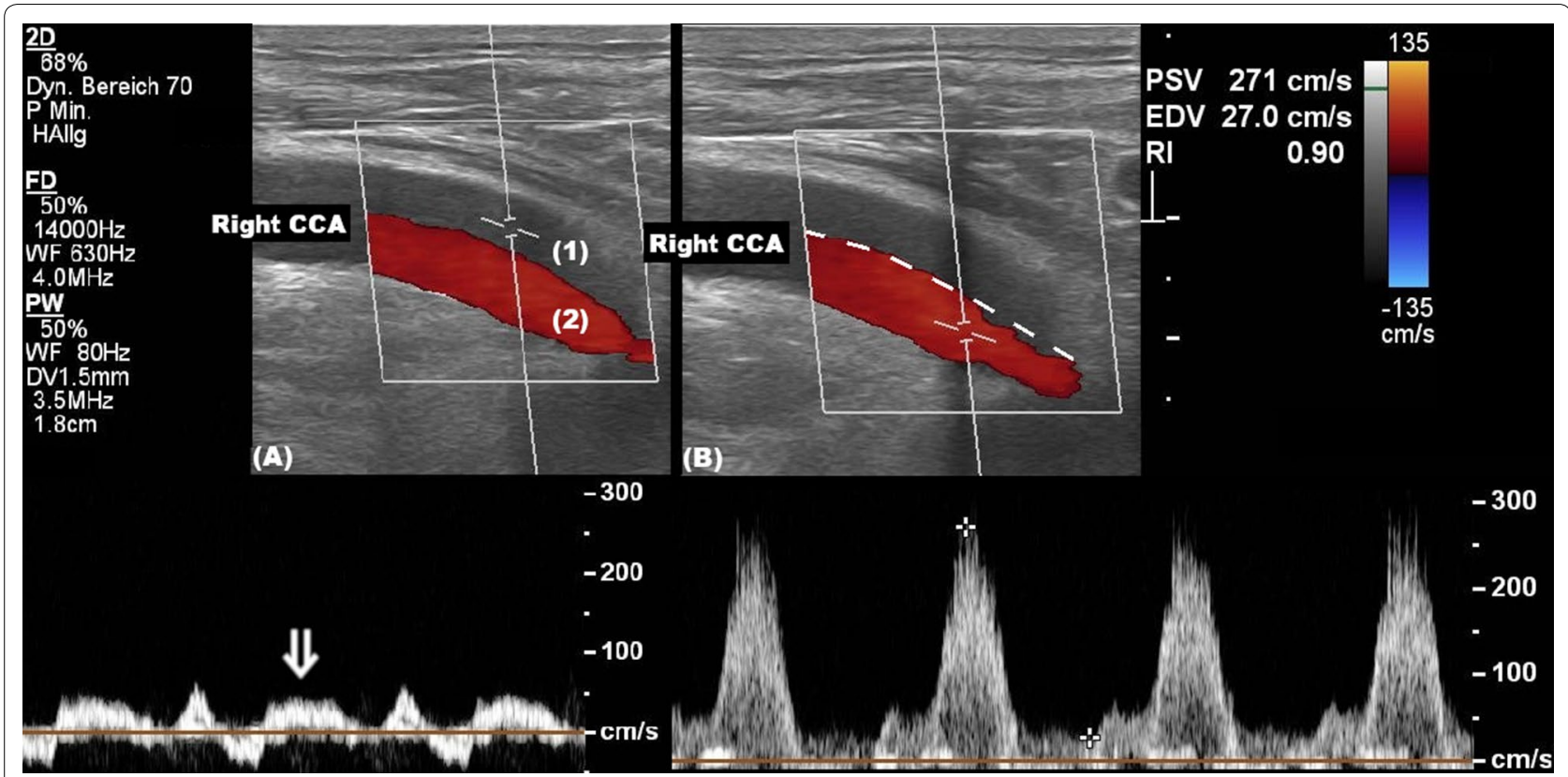

Fig. 2 Duplex sonography of the right common carotid artery. Note the dissection membrane (dashed line) separating two distinct lumina, each with different flow profiles. The false lumen (1) exhibits systolic and diastolic orthograde flow (arrow). The true lumen (2) also displays orthograde flow, but is narrowed to a luminal stenosis of $50 \%$ (peak systolic velocity $271 \mathrm{~cm} / \mathrm{s}$, reference range $29-178 \mathrm{~cm} / \mathrm{s}$ [2])

\section{Conclusion}

This case study demonstrates the effective use of bedside carotid ultrasound to diagnose carotid artery dissection due to extension of aortic dissection (CAEAD). This enabled rapid decision-making in regards to further medical management.

\section{Supplementary information}

Supplementary information accompanies this paper at https://doi. org/10.1186/s13089-019-0147-2.

Additional file 1: Video S1. B-mode ultrasound of the right common carotid artery, examined continuously, starting in the proximal segment and moving the transducer distally. (A) Transverse view. (B) Sagittal view. Note the dissection membrane, a linear and mobile hyperechogenic structure, extending from the proximal CCA to the distal ICA, separating the false (1) and true (2) lumina.

\section{Abbreviations}

AD: aortic dissection; CAEAD: carotid artery extension of aortic dissections; CCA: common carotid artery; CTA: computed tomography angiography; DSA: digital subtraction angiography; ECA: external carotid artery; ICA: internal carotid artery; ICU: intensive care unit; MRA: magnetic resonance angiography.

\section{Acknowledgements}

The authors thank Julia Wittlinger (Eberhard-Karls University of Tübingen, Department for Neurology) for her technical assistance.

\section{Authors' contributions}

$\mathrm{CB}$ and SP performed ultrasound imaging and contributed to data collection and analysis. CB drafted the manuscript. SP reviewed and edited the manuscript. Both authors read and approved the final manuscript.

\section{Funding}

We acknowledge support by the Deutsche Forschungsgemeinschaft and Open Access Publishing Fund of University of Tübingen.

\section{Availability of data and materials}

All data generated or analyzed during this study are included in this published article and its additional files.

\section{Ethics approval and consent to participate} Not applicable.

\section{Consent for publication}

Written informed consent was obtained from the patient.

\section{Competing interests}

The authors declare that they have no competing interests.

\section{Author details}

${ }^{1}$ Department of Neurology and Epileptology, and Hertie-Institute for Clinical Brain Research, Eberhard-Karls University of Tübingen, Hoppe-Seyler-Straße 3, 72076 Tübingen, Germany. ${ }^{2}$ Department of Neurology and Stroke, and Hertie-Institute for Clinical Brain Research, Eberhard-Karls University of Tübingen, Hoppe-Seyler-Straße 3, 72076 Tübingen, Germany.

Received: 20 June 2019 Accepted: 24 November 2019

Published online: 02 December 2019 


\section{References}

1. Laser A, Drucker CB, Harris DG et al (2017) Management and outcomes of carotid artery extension of aortic dissections. J Vasc Surg 66(2):445-453

2. Blackshear WM, Phillips DJ, Chikos PM, Harley JD, Thiele BL, Strandness DE (1980) Carotid artery velocity patterns in normal and stenotic vessels. Stroke 11(1):67-71

3. Baumgartner RW (2006) Handbook on neurovascular ultrasound. Front Neurol Neurosci. 21:70-84

4. Provenzale JM, Sarikaya B (2009) Comparison of test performance characteristics of MRI, MR angiography, and CT angiography in the diagnosis of carotid and vertebral artery dissection: a review of the medical literature. AJR 193(4):1167-1174

5. Brott TG, Halperin JL, Abbara S, Bacharach JM, Barr JD, Bush RL et al (2011) ASA/ACCF/AHA/AANN/AANS/ACR/ASNR/CNS/SAIP/SCAI/SIR/SNIS/SVM/ SVS guideline on the management of patients with extracranial carotid and vertebral artery disease. A report of the American College of Cardiology Foundation/American Heart Association Task Force on Practice Guidelines, and the American Stroke Association, American Association of Neuroscience Nurses, American Association of Neurological Surgeons,
American College of Radiology, American Society of Neuroradiology, Congress of Neurological Surgeons, Society of Atherosclerosis Imaging and Prevention, Society for Cardiovascular Angiography and Interventions, Society of Interventional Radiology, Society of Neurolnterventional Surgery, Society for Vascular Medicine, and Society for Vascular Surgery. Circulation 124(4):e54-e130

6. Hakimi R, Sivakumar S (2019) Imaging of carotid dissection. Curr Pain Headache Rep 23(1):2. https://doi.org/10.1007/s11916-019-0741-9

7. Clevert DA, Jung EM, Johnson T, Kubale R, Rupp N, Schoenberg SO, Reiser M (2007) Cervical artery dissection: improved diagnosis by B-Flow ultrasound. Clin Hemorheol Microcirc. 36(2):141-153

8. Jia L, Wang H, Gao Y, Liu H, Yu K (2016) High incidence of adverse events during intra-hospital transport of critically ill patients and new related risk factors: a prospective, multicenter study in China. Crit Care 20:12

\section{Publisher's Note}

Springer Nature remains neutral with regard to jurisdictional claims in published maps and institutional affiliations.

\section{Submit your manuscript to a SpringerOpen ${ }^{\circ}$ journal and benefit from:}

- Convenient online submission

- Rigorous peer review

- Open access: articles freely available online

- High visibility within the field

- Retaining the copyright to your article

Submit your next manuscript at $\boldsymbol{\nabla}$ springeropen.com 